\title{
Inferring flare loop parameters with measurements of standing sausage modes
}

\author{
Ming-Zhe Guo ${ }^{1,2} \cdot$ Shao-Xia Chen ${ }^{1} \cdot \mathrm{Bo} \mathrm{Li}^{1} \cdot$ \\ Li-Dong $\mathrm{Xia}^{1} \cdot$ Hui $\mathrm{Yu}^{1}$
}

(C) Springer $\bullet \bullet \bullet \bullet$

\begin{abstract}
Standing fast sausage modes in flare loops were suggested to account for a considerable number of quasi-periodic pulsations (QPPs) in the light curves of solar flares. This study continues our investigation into the possibility to invert the measured periods $P$ and damping times $\tau$ of sausage modes to deduce the transverse Alfvén time $R / v_{\mathrm{Ai}}$, density contrast $\rho_{\mathrm{i}} / \rho_{\mathrm{e}}$, and the steepness of the density distribution transverse to flare loops. A generic dispersion relation (DR) governing linear sausage modes is derived for pressureless cylinders where density inhomogeneity of arbitrary form takes place within the cylinder. We show that in general the inversion problem is under-determined for QPP events where only a single sausage mode exists, be the measurements spatially resolved or unresolved. While $R / v_{\mathrm{Ai}}$ can be inferred to some extent, the range of possible steepness parameters may be too broad to be useful. However, for spatially resolved measurements where an additional mode is present, it is possible to deduce self-consistently $\rho_{\mathrm{i}} / \rho_{\mathrm{e}}$, the profile steepness, and the internal Alfvén speed $v_{\mathrm{Ai}}$. We show that at least for a recent QPP event that involves a fundamental kink mode in addition to a sausage one, flare loop parameters are well constrained, even if the specific form of the transverse density distribution remains unknown. We conclude that spatially resolved, multi-mode QPP measurements need to be pursued for inferring flare loop parameters.
\end{abstract}

Keywords: Coronal Seismology; Magnetic fields, Corona; Waves, Magnetohydrodynamic

$凶 \mathrm{Bo} \mathrm{Li}$

bbl@sdu.edu.cn

1 Shandong Provincial Key Laboratory of Optical Astronomy and Solar-Terrestrial Environment, Institute of Space Sciences, Shandong University, Weihai, 264209, China

2 CAS Key Laboratory of Geospace Environment, University of Science \& Technology of China, Chinese Academy of Sciences, Hefei 230026, China 


\section{Introduction}

There is now ample evidence for the existence of low-frequency waves and oscillations in the structured solar atmosphere (for recent reviews, see e.g., Nakariakov and Verwichte, 2005; Banerjee et al., 2007; Roberts, 2008; De Moortel and Nakariakov, 2012; Liu and Ofman, 2014). When combined with magnetohydrodynamic (MHD) theory, the measured wave parameters allow one to infer the solar atmospheric parameters that are difficult to measure directly. This practice was originally proposed for the solar corona (Roberts. Edwin, and Benz 1984, see also Rosenberg 1970, Uchida 1970, Zaitsev and Stepanov 1975), but has been extended to spicules (e.g., Zaqarashvili and Erdélyi, 2009), prominences (e.g., Arregui, Oliver, and Ballester, 2012), magnetic pores (e.g., Morton et al., 2011), and various structures in the chromosphere (e.g., Jess et al., 2009; Morton et al., 2012), to name but a few. Compared with sausage waves (with azimuthal wavenumber $m=0$ ), kink waves (with $m=1$ ) have received more attention, presumably due to their ubiquity in the solar atmosphere (e.g., Nakariakov et al., 1999; Aschwanden et al., 1999; Tomczyk and McIntosh, 2009; Kupriyanova, Melnikov, and Shibasaki, 2013). However, recent observations indicated that sausage waves abound as well (e.g., Nakariakov, Melnikov, and Reznikova, 2003; Morton et al., 2012; Grant et al., 2015; Moreels et al., 2015). In addition, standing sausage modes in flare loops were shown to be important for interpreting a considerable fraction of quasi-periodic pulsations (QPPs) in the lightcurves of solar flares (see Nakariakov and Melnikov, 2009, for a recent review).

A theoretical understanding of fast sausage waves supported by magnetized cylinders is crucial for their seismological applications. For this purpose, the transverse density distribution is usually idealized as being in a step-function (top-hat) form, characterized by the internal $\left(\rho_{\mathrm{i}}\right)$ and external $\left(\rho_{\mathrm{e}}\right)$ values (e.g., Meerson, Sasorov, and Stepanov, 1978; Spruit, 1982; Edwin and Roberts, 1983; Cally, 1986; Kopylova et al., 2007; Vasheghani Farahani et al., 2014). In a low- $\beta$ environment such as the solar corona, two regimes are known to exist, depending on the longitudinal wavenumber $k$ (e.g., Spruit, 1982). When $k$ exceeds some critical $k_{\mathrm{c}}$, the trapped regime arises whereby the sausage wave energy is well confined to the cylinder. On the contrary, if $k<k_{\mathrm{c}}$, then the leaky regime results and fast sausage waves experience apparent temporal damping by emitting their energy into the surrounding fluid. Furthermore, the $k$-dependence of the periods $P$ and damping times $\tau$ of leaky waves disappears when $k$ is sufficiently small (e.g., Kopylova et al., 2007; Vasheghani Farahani et al., 2014). Let $R$ denote the cylinder radius, and $v_{\text {Ai }}$ denote the internal Alfvén speed. In the long-wavelength limit $(k \rightarrow 0), P$ is found to be primarily determined by the transverse Alfvén transit time $R / v_{\mathrm{Ai}}$, while the ratio $\tau / P$ is largely proportional to the density contrast $\rho_{\mathrm{i}} / \rho_{\mathrm{e}}$ (Kopylova et al., 2007). This then enables one to employ the measured $P$ and $\tau$ to deduce $\rho_{\mathrm{i}} / \rho_{\mathrm{e}}$ and $R / v_{\mathrm{Ai}}$, with the latter carrying important information on the magnetic field strength in flare loops.

Evidently, there is no reason to expect that the density distribution across magnetic cylinders is in a step-function fashion. This has stimulated a series of studies to examine the properties of fast sausage waves in magnetized cylinders with a continuous transverse density profile by either proceeding analytically 
with an eigen-mode analysis (Edwin and Roberts, 1988; Lopin and Nagorny, 2014, 2015) or numerically solving the linearized MHD equations as an initialvalue problem (Nakariakov, Hornsey, and Melnikov, 2012; Chen et al., 2015a). Many features in the step-function case, the $k$-dependence in particular, were found to survive. However, the period $P$ (Nakariakov, Hornsey, and Melnikov, 2012) and damping time $\tau$ (Chen et al., 2015a) may be sensitive to yet another parameter, namely the steepness or equivalently the lengthscale of the transverse density inhomogeneity. Note that the steepness is crucial in determining such coronal heating mechanisms as resonant absorption (e.g., Hollweg and Yang, 1988; Goossens, Andries, and Aschwanden, 2002; Ruderman and Roberts, 2002) and phase mixing (Heyvaerts and Priest, 1983). There is then an obvious need to employ the measured $P$ and $\tau$ of sausage modes to infer the profile steepness, in much the same way that kink modes were employed (Arregui et al., 2007; Goossens et al., 2008; Soler et al., 2014). This was undertaken by Chen et al. (2015b, hereafter paper I), based on an analytical dispersion relation (DR) governing linear fast sausage waves in cylinders with a rather general transverse density distribution. The only requirement was that this profile can be decomposed into a uniform cord, a uniform external medium, and a transition layer connecting the two. However, this layer can be of arbitrary width and the profile therein can be in arbitrary form.

The aim of the present study is to extend the analysis in paper I in the following aspects. First, we will remove the restriction for the transverse density profile to involve a uniform cord, thereby enabling the analysis to be applicable to a richer variety of density distributions. Second, when validating the results from this eigenmode analysis, we employ an independent approach by solving the time-dependent version of linear MHD equations. We will detail these timedependent computations pertinent to the afore-mentioned transverse density profile. Third, we will extend the seismological applications in paper I to QPP events that involve both kink and sausage modes. To illustrate the scheme for inverting multi-mode measurements, paper I adopted the analytical expressions for the kink mode period and damping time in the thin-tube-thin-boundary (TTTB) limit as given by Goossens et al. (2008). In this study we replace the TTTB expressions with a self-consistent, linear, resistive MHD computation. This is necessary given that flare loops tend not to be thin (Aschwanden, Nakariakov, and Melnikov, 2004), and it is not safe to assume a priori that the density inhomogeneity takes place in a thin transition layer. Fourth, in connection with the third point, we take this opportunity to provide a rather detailed examination of resonantly damped kink modes in cylinders with transverse density profiles in question.

This manuscript is organized as follows. Section 2 presents the necessary equations, the derivation of the analytic DR in particular. The behavior of sausage waves in nonuniform cylinders and its applications to QPP events are then presented in Sect. 3. Finally, Sect. 4 summarizes the present study. 


\section{Mathematical Formulation}

\subsection{Derivation of the Dispersion Relation}

Appropriate for the solar corona, we adopt ideal, cold (zero- $\beta$ ) MHD to describe fast sausage waves. The magnetic loops hosting these waves are modeled as straight cylinders with radius $R$ aligned with a uniform magnetic field $\boldsymbol{B}=$ $B \hat{z}$, where a standard cylindrical coordinate system $(r, \theta, z)$ is adopted. The equilibrium density is assumed to be a function of $r$ only and of the form

$$
\rho(r)=\left\{\begin{array}{cc}
\rho_{\mathrm{i}}\left[1-\left(1-\frac{\rho_{\mathrm{e}}}{\rho_{\mathrm{i}}}\right) f(r)\right], & 0 \leq r<R \\
\rho_{\mathrm{e}}, & r>R,
\end{array}\right.
$$

where $f(r)$ is some arbitrary function that increases smoothly from 0 at $r=0$ to unity when $r=R$. Furthermore, $\rho_{\mathrm{i}}$ and $\rho_{\mathrm{e}}$ denote the densities at the cylinder axis and in the external medium, respectively. The corresponding Alfvén speeds follow from the definition $v_{\mathrm{Ai}, \mathrm{e}}=B / \sqrt{4 \pi \rho_{\mathrm{i}, \mathrm{e}}}$.

It suffices to briefly outline the mathematical approach for establishing the pertinent dispersion relation (DR), since this approach has been detailed in paper I. To start, we specialize to axisymmetric sausage perturbations, and Fourieranalyze any perturbation $\delta f(r, z, t)$ as

$$
\delta f(r, z, t)=\operatorname{Re}\{\tilde{f}(r) \exp [-i(\omega t-k z)]\}
$$

It then follows from the linearized, ideal, cold MHD equations that the Fourier amplitudes of the transverse Lagrangian displacement $\left(\tilde{\xi}_{r}\right)$ and Eulerian perturbation of total pressure $\left(\tilde{p}_{\mathrm{T}}\right)$ are governed by Eqs. (6) and (7) in paper I, respectively. Now that sausage waves do not resonantly couple to torsional Alfvén waves for the configuration we examine, one may employ regular series expansions about $y \equiv r-R / 2=0$ to express $\tilde{\xi}_{r}$ and $\tilde{p}_{\mathrm{T}}$ in the nonuniform portion of the density distribution. Further requiring that sausage waves do not disturb the cylinder axis $\left(\tilde{\xi}_{r}=0\right.$ at $\left.r=0\right)$, and employing the conditions for $\tilde{\xi}_{r}$ and $\tilde{p}_{\mathrm{T}}$ to be continuous at the interface $r=R$, one finds that the DR can be expressed as

$$
\begin{aligned}
& \frac{\frac{\mu_{\mathrm{e}} R H_{0}^{(1)}\left(\mu_{\mathrm{e}} R\right)}{H_{1}^{(1)}\left(\mu_{\mathrm{e}} R\right)} \tilde{\xi}_{1}(R / 2)-\tilde{\xi}_{1}(R / 2)-R \tilde{\xi}_{1}^{\prime}(R / 2)}{\tilde{\xi}_{1}(-R / 2)} \\
= & \frac{\frac{\mu_{\mathrm{e}} R H_{0}^{(1)}\left(\mu_{\mathrm{e}} R\right)}{H_{1}^{(1)}\left(\mu_{\mathrm{e}} R\right)} \tilde{\xi}_{2}(R / 2)-\tilde{\xi}_{2}(R / 2)-R \tilde{\xi}_{2}^{\prime}(R / 2)}{\tilde{\xi}_{2}(-R / 2)} .
\end{aligned}
$$


Here $H_{n}^{(1)}$ denotes the $n$ th-order Hankel function of the first kind, and $\mu_{\mathrm{e}}$ is defined by

$$
\mu_{\mathrm{e}}^{2}=\frac{\omega^{2}}{v_{\mathrm{Ae}}^{2}}-k^{2} \quad\left(-\frac{\pi}{2}<\arg \mu_{\mathrm{e}} \leq \frac{\pi}{2}\right) .
$$

Furthermore,

$$
\tilde{\xi}_{1}(y)=\sum_{n=0}^{\infty} a_{n} y^{n} \text { and } \tilde{\xi}_{2}(y)=\sum_{n=0}^{\infty} b_{n} y^{n}
$$

are two linearly independent solutions for $\tilde{\xi}_{r}$ in the portion $r<R$. Without loss of generality, we choose

$$
a_{0}=R, \quad a_{1}=0, \quad b_{0}=0, \quad b_{1}=1 .
$$

The rest of the coefficients $a_{n}$ and $b_{n}$ can be found by replacing $R$ with $R / 2$ in Eq. (11) in paper I, and contain the information on the density distribution. Finally, the prime ' denotes the derivative of $\tilde{\xi}_{1,2}$ with respective to $y$.

Before proceeding, we note that a series-expansion-based approach was recently adopted by Soler et al. (2013) to treat wave modes in transversally nonuniform cylinders where the azimuthal wavenumber $m$ is allowed to be arbitrary. A comparison between that approach and ours is detailed in Appendix $\mathrm{C}$. where we show that both approaches yield identical results for trapped sausage modes $(m=0)$. While our approach seems more appropriate to describe leaky sausage modes, we stress that a singular expansion as employed by Soler et al. (2013) is necessary to treat wave modes with $m \neq 0$.

\subsection{Method of solution}

Throughout this study, we focus on standing sausage modes by restricting longitudinal wavenumbers $(k)$ to be real, but allowing angular frequencies $(\omega)$ to be complex-valued $\left(\omega=\omega_{\mathrm{R}}+i \omega_{\mathrm{I}}\right)$. In addition, we focus on fundamental modes, namely those with $k=\pi / L$ where $L$ is the loop length. In practice, we start with prescribing an $f(r)$, and then solve Eq. (3) by truncating the infinite series expansion (Eq. [5) to retain terms with $n$ up to $N=101$. Using an even larger $N$ leads to no appreciable difference. It should be noted that $\omega$ in units of $v_{\mathrm{Ai}} / R$ depends only on the combination $\left[f(r), k R, \rho_{\mathrm{i}} / \rho_{\mathrm{e}}\right]$. The corresponding period $P$ and damping time $\tau$ follow from the definitions $P=2 \pi / \omega_{\mathrm{R}}$ and $\tau=1 /\left|\omega_{\mathrm{I}}\right|$.

For validation purposes, we also obtain $\omega$ as a function of $k$ in a way independent from this eigen-mode analysis. This is done by solving the time-dependent equation governing the transverse velocity perturbation $\delta v_{r}(r, z, t)$ as an initialvalue problem. For given combinations of $\left[f(r), k R, \rho_{\mathrm{i}} / \rho_{\mathrm{e}}\right]$, one may find the periods and damping times of sausage modes by analyzing the temporal evolution of the perturbation signals (see Appendix $\mathrm{A}$ for details). As will be shown by Fig. 2 , the values of $P$ and $\tau$ derived from the two independent approaches are in close agreement. However, numerically solving the analytical DR is much less computationally expensive. On top of that, the values of $\tau$ for heavily damped modes can be readily found, whereas the perturbation signals in time-dependent computations decay too rapidly to allow a proper determination of $\tau$. 


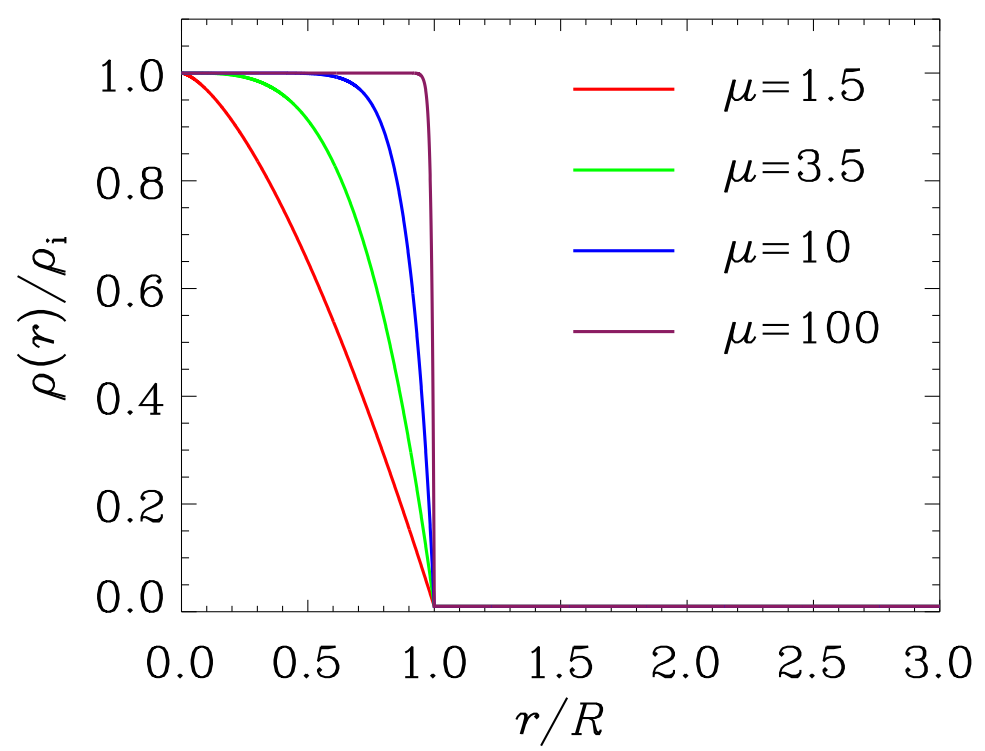

Figure 1. Transverse equilibrium density profiles as a function of $r$ for different steepness parameters $\mu$ as labeled. Here the density contrast $\rho_{\mathrm{i}} / \rho_{\mathrm{e}}$ is chosen to be 100 for illustration purposes.

\section{Numerical Results}

It is impossible to exhaust the possible prescriptions for $f(r)$. We therefore focus on one choice, namely

$$
f(r)=\left(\frac{r}{R}\right)^{\mu}
$$

where $\mu$ is positive. The density profiles with a number of different $\mu$ are shown in Fig. 1] where $\rho_{\mathrm{i}} / \rho_{\mathrm{e}}$ is chosen to be 100 for illustration purposes. Evidently, the profile becomes increasingly steep as $\mu$ increases and approaches a step-function form when $\mu$ approaches infinity. This makes it possible to investigate the effect of profile steepness by examining the $\mu$-dependence of the numerical results. In addition, for fundamental modes with $k=\pi / L$, the dependence on $k R$ is translated into that on the length-to-radius ratio $L / R$.

\subsection{Behavior of sausage waves in nonuniform tubes}

Figure 2 presents the dependence on $L / R$ of the period $P$ and damping time $\tau$ for a series of $\mu$ values as labeled. For illustration purposes, the density ratio $\rho_{\mathrm{i}} / \rho_{\mathrm{e}}$ is taken to be 100 . The dash-dotted line in Fig. 2 a represents $P=2 L / v_{\mathrm{Ae}}$, and separates trapped (to its left) from leaky (right) modes. The solid curves represent the results from solving the analytical DR (Eq. 3), whereas the circles represent those obtained with the initial-value-problem approach. A close agreement between the curves and circles is clear, thereby validating the DR.

Figure 2 a indicates that the wave period $P$ increases monotonically with $L / R$ in the trapped regime, and rapidly settles to some asymptotic value in the leaky 

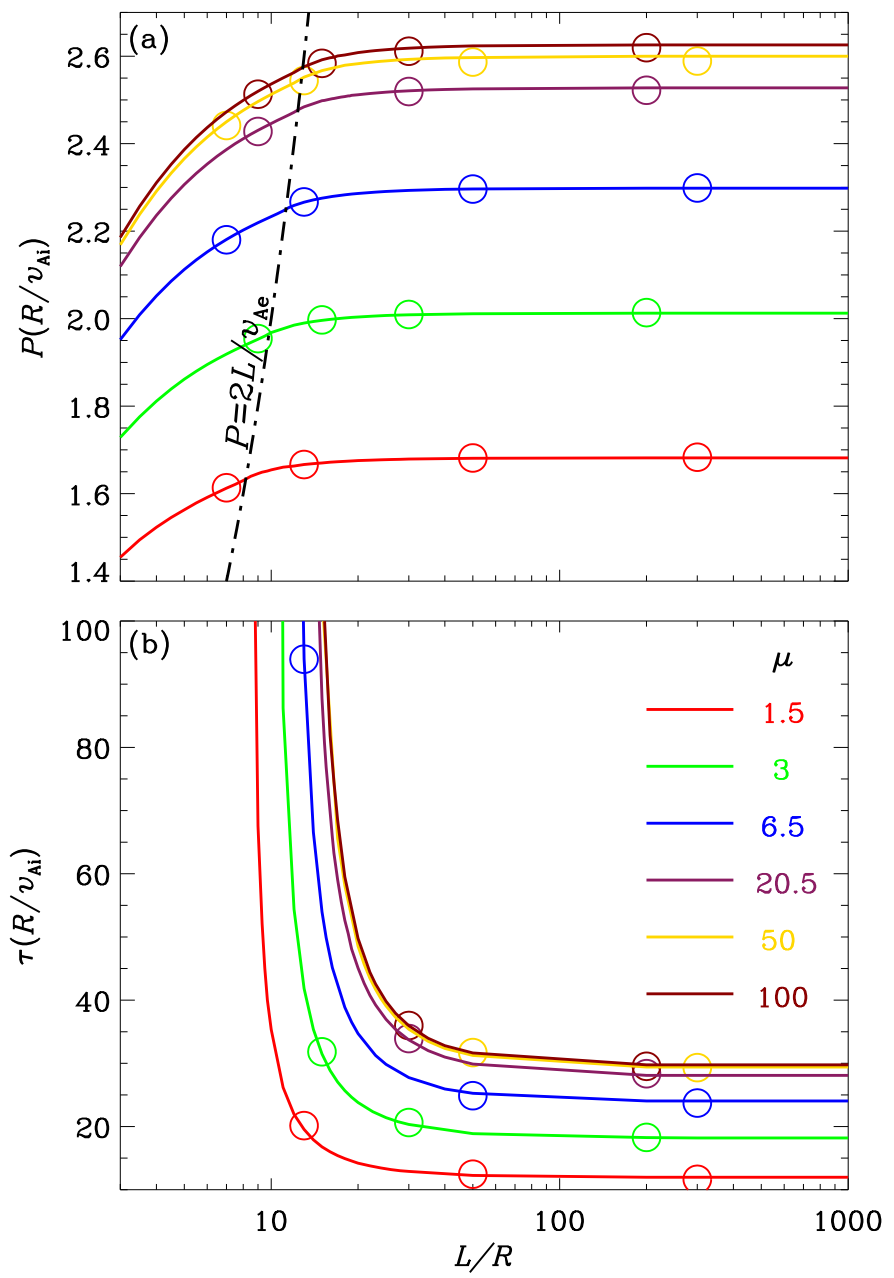

Figure 2. Dependence on length-to-radius ratio $L / R$ of (a) periods $P$ and (b) damping times $\tau$ of fundamental sausage modes. A number of density profiles with different $\mu$ are examined as labeled. The black dash-dotted line in (a) represents $P=2 L / v_{\mathrm{Ae}}$ and separates the trapped (to its left) from leaky (right) regimes. The open circles represent the values obtained by solving Eq. (12) with an initial-value-problem approach, which is independent from the eigen-mode analysis presented in the text. The density contrast $\rho_{\mathrm{i}} / \rho_{\mathrm{e}}$ is chosen to be 100 .

one. Likewise, Fig. 20 shows that, being identically infinite in the trapped regime, the damping time $\tau$ also experiences saturation for sufficiently large $L / R$. In addition, both $P$ and $\tau$ increase substantially with increasing $\mu$ at a given $L / R$. We note that while the tendency for $P$ to be larger for steeper density profiles agrees with the study by Nakariakov, Hornsey, and Melnikov (2012), it does not hold in general. As a matter of fact, Fig. 3 in paper I shows that the opposite occurs for some different profile prescriptions. This means that the largely unknown specific form of the transverse density distribution plays an important role in 
determining the dispersive properties of sausage modes. Consequently, when the period and damping time of sausage modes are seismologically exploited, the uncertainty in specifying the density profile needs to be considered.

\subsection{Applications to spatially unresolved QPP observations}

In essence, Fig. 2 indicates that the period $P$ and damping time $\tau$ of sausage modes can be formally expressed as

$$
\begin{aligned}
& P_{\text {saus }}=\frac{R}{v_{\mathrm{Ai}}} F_{\text {saus }}\left(\frac{L}{R}, \mu, \frac{\rho_{\mathrm{i}}}{\rho_{\mathrm{e}}}\right), \\
& \frac{\tau_{\text {saus }}}{P_{\text {saus }}}=G_{\text {saus }}\left(\frac{L}{R}, \mu, \frac{\rho_{\mathrm{i}}}{\rho_{\mathrm{e}}}\right) .
\end{aligned}
$$

Note that the damping-time-to-period ratio $\tau / P$ is adopted here instead of $\tau$ itself, the reason being that $\tau / P$ does not depend on $R / v_{\mathrm{Ai}}$. Furthermore, the $L / R$-dependence disappears for cylinders with large enough $L / R$.

Let us first consider the applications of Eqs. (8) and (9) to spatially unresolved QPP events, for which only $P$ and/or $\tau$ can be regarded known. However, the information is missing on both the physical parameters $\left[v_{\mathrm{Ai}}, \mu, \rho_{\mathrm{i}} / \rho_{\mathrm{e}}\right]$ and geometrical parameters $[L, R]$. If a trapped sausage mode is responsible for causing a QPP event as happens when the signals do not show clear damping, then only Eq. (8) is relevant. This means that any point on a 3-dimensional (3D) hypersurface in the $4 \mathrm{D}$ space formed by $\left[R / v_{\mathrm{Ai}}, L / R, \mu, \rho_{\mathrm{i}} / \rho_{\mathrm{e}}\right]$ is possible to reproduce the measured $P$. Even if the signals in a QPP event are temporally decaying, the range of possible parameters that can reproduce the measured $P$ and $\tau$ is still too broad to be useful: a 2D surface in the 4D parameter space results. The situation improves if one can assume that the flare loops hosting sausage modes are sufficiently thin such that the $L / R$-dependence drops out. Equations (8) and (9) then suggest that for trapped (leaky) modes, one can deduce a $2 \mathrm{D}$ surface (1D curve) in the $3 \mathrm{D}$ space formed by $\left[R / v_{\mathrm{Ai}}, \mu, \rho_{\mathrm{i}} / \rho_{\mathrm{e}}\right]$. We note that the idea for deriving $1 \mathrm{D}$ inversion curves was first introduced by Arregui et al. (2007) and later explored in e.g., Goossens et al. (2008); Soler et al. (2014). While resonantly damped kink modes were examined therein, the same idea applies also to leaky sausage modes in thin cylinders, the only difference being that the transverse Alfvén time $\left(R / v_{\mathrm{Ai}}\right)$ replaces the longitudinal one $\left(L / v_{\mathrm{Ai}}\right)$.

Figure 3 presents the $1 \mathrm{D}$ curve and its projections (the dashed lines) onto various planes in the $\left[R / v_{\mathrm{Ai}}, \mu, \rho_{\mathrm{i}} / \rho_{\mathrm{e}}\right]$ space, using the QPP event reported in McLean and Sheridan (1973) as an example. For this event, the oscillation period is 4.3 secs, and the damping-time-to-period ratio is 10 . Table 1 presents a set of values read from the solid curve in Fig. 3. One can see that among the parameters to infer, the transverse Alfvén time $R / v_{\mathrm{Ai}}$ and density ratio $\rho_{\mathrm{i}} / \rho_{\mathrm{e}}$ can be somehow constrained. To be specific, the pair $\left[R / v_{\mathrm{Ai}}, \rho_{\mathrm{i}} / \rho_{\mathrm{e}}\right]$ reads [2.94 secs, 182] when $\mu=1$, and reads [1.64 secs, 88.2] when $\mu=100$. However, the steepness parameter $\mu$ is difficult to constrain, since its possible range is too broad. This agrees with paper I where we concluded that for spatially unresolved 


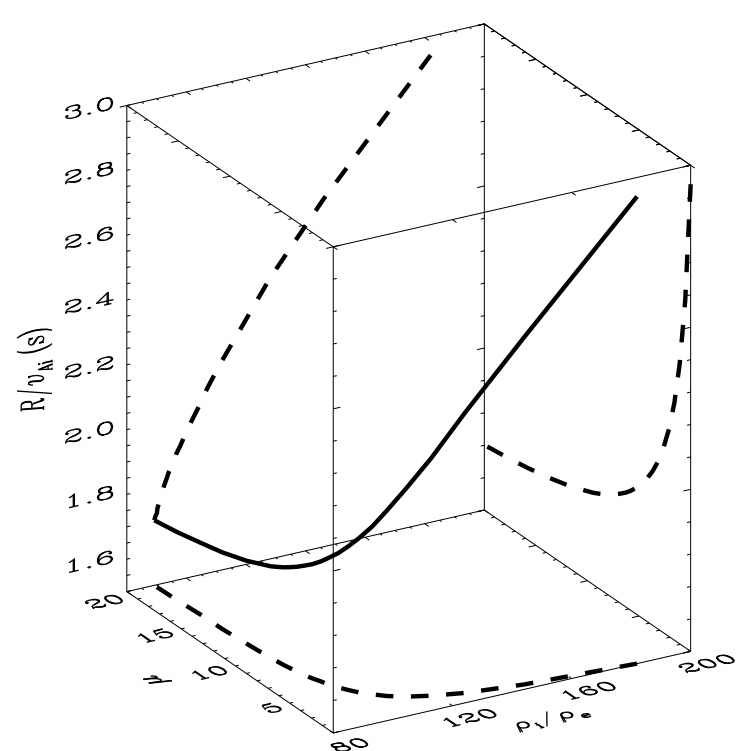

Figure 3. Inversion curve (the solid line) and its projections (dashed) in the three-dimensional parameter space spanned by $\left[\mu, \rho_{\mathrm{i}} / \rho_{\mathrm{e}}, R / v_{\mathrm{Ai}}\right]$. All points along this curve are equally possible to reproduce the quasi-periodic-pulsation event reported in McLean and Sheridan (1973), where the oscillation period is 4.3 secs, and the damping-time-to-period ratio is 10 .

QPPs, the transverse Alfvén time is the best constrained, whereas the steepness (the length of the transition layer in units of loop radius $l / R$ in that paper) corresponds to the other extreme.

Table 1. Values of $\left[\mu, \rho_{\mathrm{i}} / \rho_{\mathrm{e}}, R / v_{\mathrm{Ai}}\right]$ deduced for the QPP event reported in McLean and Sheridan (1973).

\begin{tabular}{|c|c|c|c|c|c|c|c|c|c|}
\hline$\mu$ & $\|$ & 2.5 & 4 & 6.5 & 9 & 12 & 20 & 60 & 100 \\
\hline$\rho_{\mathrm{i}} / \rho_{\mathrm{e}}$ & || 182.0 & 118.3 & 104.0 & 95.8 & 92.5 & 91.0 & 89.2 & 88.2 & 88.2 \\
\hline$R / v_{\mathrm{Ai}}(\mathrm{secs})$ & || 2.94 & 2.23 & 2.02 & 1.87 & 1.80 & 1.76 & 1.7 & 1.65 & 1.64 \\
\hline
\end{tabular}

One may then ask how to make sense of this seismological inversion. To this end, we may compare our results with what one finds with the DR for a stepfunction density profile (Eq. 18 in paper I). Noting that the $\mu$-dependence no longer exists in the step-function case, one finds with the measured $P$ and $\tau$ that $\left[R / v_{\mathrm{Ai}}, \rho_{\mathrm{i}} / \rho_{\mathrm{e}}\right]=[1.62 \mathrm{secs}, 88.2]$. As expected, this agrees well with what we found for large $\mu$. However, it differs substantially from the results for small $\mu$. From this we conclude that, although simple and straightforward, the practice for deducing $\left[R / v_{\mathrm{Ai}}, \rho_{\mathrm{i}} / \rho_{\mathrm{e}}\right]$ using the DR for step-function profiles is subject to substantial uncertainty if one takes account of the uncertainties in prescribing the transverse density structuring. In particular, it may substantially underestimate $R / v_{\mathrm{Ai}}$. This uncertainty will be carried over to the deduced values 
of the Alfvén speed and consequently the magnetic field strength, provided that one can further estimate the loop radius $R$ and internal density $\rho_{\mathrm{i}}$.

\subsection{Applications to spatially resolved QPP observations}

Now move on to the seismological applications of Eqs. (8) and (9) to spatially resolved QPP events. In this case the geometrical parameters $L$ and $R$ can be considered known, and only the combination of $\left[v_{\mathrm{Ai}}, \mu, \rho_{\mathrm{i}} / \rho_{\mathrm{e}}\right]$ remains to be deduced. It then follows that if a trapped (leaky) mode is presumably the cause of a QPP event, the measured period $P(P$ together with the damping time $\tau$ ) allows a 2D surface (1D curve) to be found in the 3D space formed by $\left[v_{\mathrm{Ai}}, \mu, \rho_{\mathrm{i}} / \rho_{\mathrm{e}}\right]$.

Something more definitive can be deduced if a QPP event involves more than just a sausage mode. Similar to paper I, let us examine the case where a fundamental kink mode exists together with a fundamental sausage one, with both experiencing temporal damping. Let $P_{\text {saus }}$ and $\tau_{\text {saus }}$ denote the period and damping time of the sausage mode, respectively. Likewise, let $P_{\text {kink }}\left(\tau_{\text {kink }}\right)$ denote the period (damping time) of the kink mode. Furthermore, let us assume that wave leakage leads to the apparent damping of the sausage mode, whereas resonant absorption is responsible for damping the kink mode. One finds that $P_{\text {kink }}$ and $\tau_{\text {kink }}$ can be formally expressed as

$$
\begin{aligned}
& P_{\text {kink }}=\frac{L}{v_{\mathrm{Ai}}} F_{\text {kink }}\left(\frac{L}{R}, \mu, \frac{\rho_{\mathrm{i}}}{\rho_{\mathrm{e}}}\right), \\
& \tau_{\text {kink }}=\frac{L}{v_{\mathrm{Ai}}} H_{\text {kink }}\left(\frac{L}{R}, \mu, \frac{\rho_{\mathrm{i}}}{\rho_{\mathrm{e}}}\right) .
\end{aligned}
$$

To establish the functions $F_{\text {kink }}$ and $H_{\text {kink }}$, we adopt the same approach as in Terradas, Oliver, and Ballester (2006). A set of linearized resistive MHD equations (Eqs. 1-5 therein) is solved for the dimensionless complex angular frequency $\left(\omega_{\text {kink }} L / v_{\mathrm{Ai}}\right)$ as an eigen-value. A uniform resistivity $\bar{\eta}$ is adopted, resulting in a magnetic Reynolds number $R_{\mathrm{m}}=v_{\mathrm{Ai}} R / \bar{\eta}$. It turns out that $\omega_{\mathrm{kink}} L / v_{\mathrm{Ai}}$ does not depend on $R_{\mathrm{m}}$ when $R_{\mathrm{m}}$ is sufficiently large, and this saturation value is taken to be the value that $\omega_{\text {kink }} L / v_{\mathrm{Ai}}$ attains with the input parameters $\left[L / R, \mu, \rho_{\mathrm{i}} / \rho_{\mathrm{e}}\right]$ (see Appendix B for details). We note that one can also establish $F_{\text {kink }}$ and $H_{\text {kink }}$ with the approach developed by Soler et al. (2013), where a less computationally costly method based on singular series expansions was employed.

With $P_{\text {saus }}, \tau_{\text {saus }}, P_{\text {kink }}$ and $\tau_{\text {kink }}$ measured, one finds that the number of equations is more than needed, since now there are only three unknowns, $v_{\mathrm{Ai}}$, $\mu$, and $\rho_{\mathrm{i}} / \rho_{\mathrm{e}}$. In practice, we consider the expression for $P_{\mathrm{kink}}$ as the redundant one, and use the rest for seismological purposes. As suggested by paper I, the kink mode period expected from Eq. (10) with the deduced parameters can be compared with the measured value. The difference between the two allows one to say, for instance, whether it is safe to identify the oscillating signals with the particular modes. In addition, this difference can also serve as an estimate of the errors of the deduced loop parameters for a given density prescription.

While seemingly fortuitous, QPP events involving multiple modes do occur (e.g., Nakariakov, Melnikov, and Reznikova, 2003; Kupriyanova, Melnikov, and 

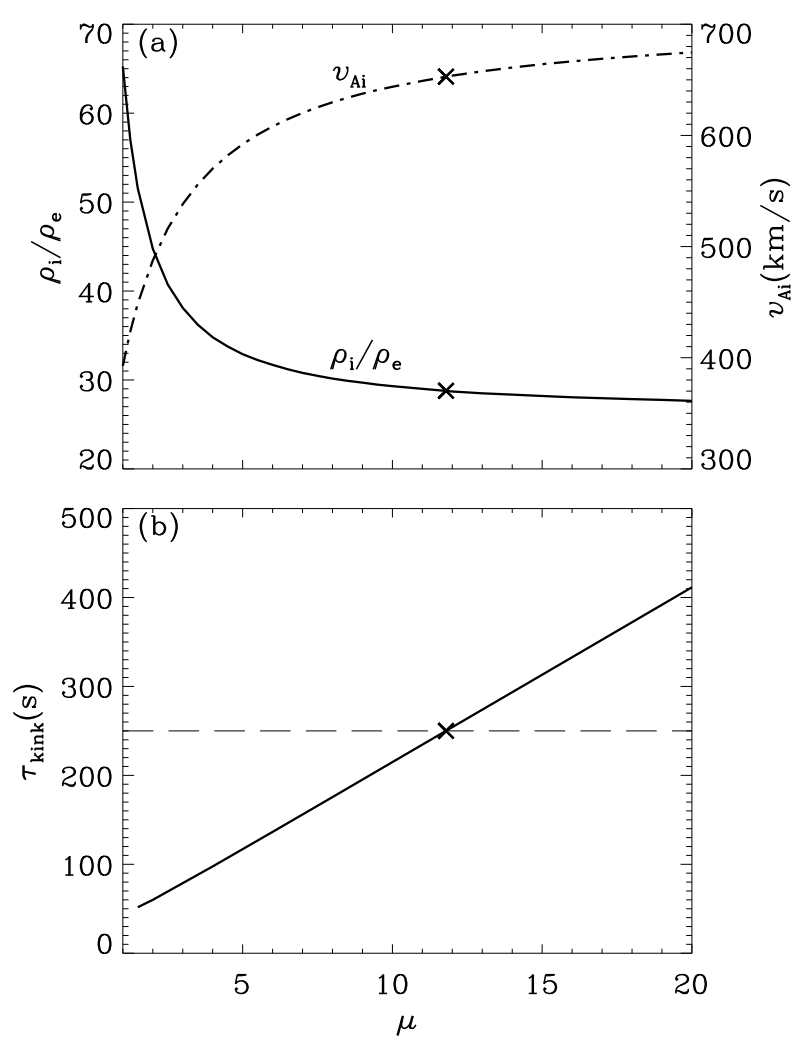

Figure 4. Illustration of the scheme for inverting the two-mode QPP event reported in Kolotkov et al. (2015). The curves in (a) are found by requiring that the damping-timeto-period ratio $\tau_{\text {saus }} / P_{\text {saus }}$ and period $P_{\text {saus }}$ for fundamental sausage modes to agree with the measured values for a series of given values of $\mu$. The solid curve in (b) represents the damping time $\tau_{\text {kink }}$ for fundamental kink modes expected with the values $\left[\mu, \rho_{\mathrm{i}} / \rho_{\mathrm{e}}, v_{\mathrm{Ai}}\right]$ given in (a). Its intersection with the horizontal dashed line, representing the measured value for $\tau_{\text {kink }}$, gives a unique combination of $\left[\mu, \rho_{\mathrm{i}} / \rho_{\mathrm{e}}, v_{\mathrm{Ai}}\right]$ labeled by the crosses.

Shibasaki, 2013; Kolotkov et al.,2015). For instance, when analyzing the multiple signals in the QPP event on 14 May 2013, Kolotkov et al. (2015) identified a fundamental fast kink mode with period $P_{\text {kink }}=100$ secs and damping time $\tau_{\text {kink }}=250$ secs together with a fundamental sausage mode with $P_{\text {saus }}=15$ secs and $\tau_{\text {saus }}=90$ secs. In addition, the flare loop hosting the two modes was suggested to be of length $L=4 \times 10^{4} \mathrm{~km}$ and radius $R=4 \times 10^{3} \mathrm{~km}$, if one takes the apparent width of the loop as the loop diameter. Now the seismological inversion is rather straightforward and involves only two steps as illustrated by Fig. 4. First, with the aid of Eq. (9), one readily derives a curve (the solid curve in Fig. $4 \mathrm{H})$ in the $\left[\mu, \rho_{\mathrm{i}} / \rho_{\mathrm{e}}\right]$ plane to be compatible with the measured $\tau_{\text {saus }} / P_{\text {saus }}$. The internal Alfvén speed $v_{\mathrm{Ai}}$ for a given pair of $\left[\mu, \rho_{\mathrm{i}} / \rho_{\mathrm{e}}\right]$ is then found with Eq. (8) to agree with the measured $P_{\text {saus }}$, yielding the dash-dotted curve. Second, one evaluates the kink mode damping time with Eq. (11) with a series of combinations $\left[v_{\mathrm{Ai}}, \mu, \rho_{\mathrm{i}} / \rho_{\mathrm{e}}\right]$, thereby finding the solid curve in Fig. $4 \mathrm{~b}$. The intersection of this solid curve with the horizontal dashed line, representing 
the measured kink mode damping time $\left(\tau_{\text {kink }}=250\right.$ secs $)$, then yields that $\mu=11.8, \rho_{\mathrm{i}} / \rho_{\mathrm{e}}=28.8$, and $v_{\mathrm{Ai}}=653 \mathrm{~km} \mathrm{~s}^{-1}$. It is worth stressing that Eq. (10) yields an expected kink mode period of 88 secs with the measured $L$ and $R$ as well as this set of deduced parameters. This is close to what was measured (100 secs), substantiating the interpretation of the long-period signal as the fundamental kink mode as done by Kolotkov et al. (2015). Alternatively, this agreement between the two values also suggests that the errors in this inversion procedure are rather moderate.

What are the uncertainties of the derived flare loop parameters? Evidently, these come entirely from the uncertainties associated with the unknown specific form of the transverse density structuring. To provide an uncertainty measure, we repeat the afore-mentioned inversion process for all four different density prescriptions in paper I, where we examined only one profile (the sine one) and adopted the TTTB approximation to describe $F_{\text {kink }}$ and $H_{\text {kink }}$. Now with the pertinent analytical DRs for sausage modes and self-consistent resistive MHD computations for kink modes, we find that the density contrast $\rho_{\mathrm{i}} / \rho_{\mathrm{e}}$ is constrained to the range from 28.4 to 31.1, and the internal Alfvén speed $v_{\mathrm{Ai}}$ lies between 594 and $658 \mathrm{~km} \mathrm{~s}^{-1}$. Interestingly, for the $\mu$-power profile examined here, the values inferred for $\rho_{\mathrm{i}} / \rho_{\mathrm{e}}$ and $v_{\mathrm{Ai}}$ also lie in these rather narrow ranges. On the other hand, the deduced $\mu$ value indicates that the density profile across the flare loop in question is rather steep, which also agrees with the ratios of the transition layer width to loop radius $(0.167 \leq l / R \leq 0.284)$ inferred with the profile prescriptions in paper I. From this we conclude that at least for the profiles examined in the present study and paper I, the uncertainties of the inferred profile steepness, density contrast, and internal Alfvén speed are relatively small.

\section{Summary}

A substantial fraction of quasi-periodic pulsations (QPPs) in the lightcurves of solar flares is attributed to sausage modes in flare loops. The present study continues our effort initiated in Chen et al. (2015b, paper I) to infer flare loop parameters with the measured periods $P$ and damping times $\tau$ of fundamental standing sausage modes supported therein. For this purpose we extended the analysis in paper I to sausage waves in nonuniform, straight, coronal cylinders with arbitrary transverse density profiles comprising a nonuniform inner portion and a uniform external medium. Working in the framework of ideal, cold, MHD, we derived an analytical dispersion relation (DR, Eq. 31) and focused on density profiles of a $\mu$-power form (Eq. 77). The dispersive properties of fundamental, standing modes were examined, together their potential for inferring flare loop parameters.

We found that $P$ and $\tau$ in units of the transverse Alfvén time $R / v_{\mathrm{Ai}}$ depend only on the density contrast $\rho_{\mathrm{i}} / \rho_{\mathrm{e}}$, length-to-radius ratio $L / R$ of coronal cylinders, and the profile steepness $\mu$. For all profiles examined in both this study and paper I, when the rest of the parameters are fixed, $P(\tau)$ in units of $R / v_{\mathrm{Ai}}$ increases (decreases) with increasing $L / R$ and tends to some saturation value when 
$L / R$ is sufficiently large. For spatially unresolved QPPs, we showed that one can at most deduce a curve in the 3 -dimensional space formed by $R / v_{\mathrm{Ai}}, \rho_{\mathrm{i}} / \rho_{\mathrm{e}}$, and $\mu$. This happens when one can assume that $L / R \gg 1$ beforehand. Applying this inversion procedure to the event reported by McLean and Sheridan (1973), we found that $R / v_{\mathrm{Ai}}$ is the best constrained, whereas the steepness parameter is the least constrained. For spatially resolved QPPs, we showed that while geometric parameters of flare loops are available, the inversion problem remains underdetermined. However, when an additional mode co-exists with the fundamental sausage mode, the full information on the unknowns, $\left[v_{\mathrm{Ai}}, \mu, \rho_{\mathrm{i}} / \rho_{\mathrm{e}}\right]$, can be inferred. In fact, in this case the inversion problem may become over-determined. Applying this idea to a recent QPP event where temporally decaying kink and sausage modes were identified, we found that $v_{\mathrm{Ai}}, \rho_{\mathrm{i}} / \rho_{\mathrm{e}}$, and the profile steepness can be constrained to rather narrow ranges.

The discussions on the limitations to our inversion procedures as presented in paper I also apply here and will not be repeated. Instead, let us stress the great potential of using multi-mode QPP measurements to determine flare loop parameters rather precisely, the internal Alfvén speed in particular. To this end, not only modes of distinct nature (e.g., a fundamental kink mode co-existing with a sausage one) are useful, modes of the same nature but with different longitudinal node numbers can also do the job. While fundamental kink modes and their harmonics have been seismologically exploited (see e.g., the review by Andries et al., 2009), serious studies using sausage modes need to be conducted.

Before closing, we note that Bayesian techniques have been successfully applied to the inference of density structuring transverse to coronal loops hosting resonantly damping kink modes (Asensio Ramos and Arregui, 2013; Arregui, Asensio Ramos, and Pascoe, 2013; Arregui, Soler, and Asensio Ramos, 2015). With such techniques, the errors in the measurements of kink mode periods and damping times can be properly propagated, and the plausibility of a density profile prescription can be assessed. When no particular prescription is favored, approaches like model-averaging can be employed to yield an evidence-averaged inference. While so far the applications of such techniques have been primarily focused on kink modes, similar ideas are expected to be equally applicable to sausage modes. For this purpose, the DRs derived here and in paper I should be useful.

Acknowledgments We thank the referee for his/her constructive comments, which helped improve this manuscript substantially. This research is supported by the 973 program 2012CB825601, National Natural Science Foundation of China (41174154, 41274176, 41274178, and 41474149), the Provincial Natural Science Foundation of Shandong via Grant JQ201212, and also by a special fund of Key Laboratory of Chinese Academy of Sciences.

\section{Appendix}

\section{A. Fast sausage modes in nonuniform cylinders: a time-dependent approach}

This section provides a detailed examination of sausage modes from an initialvalue-problem perspective. We note that similar studies were carried out for 


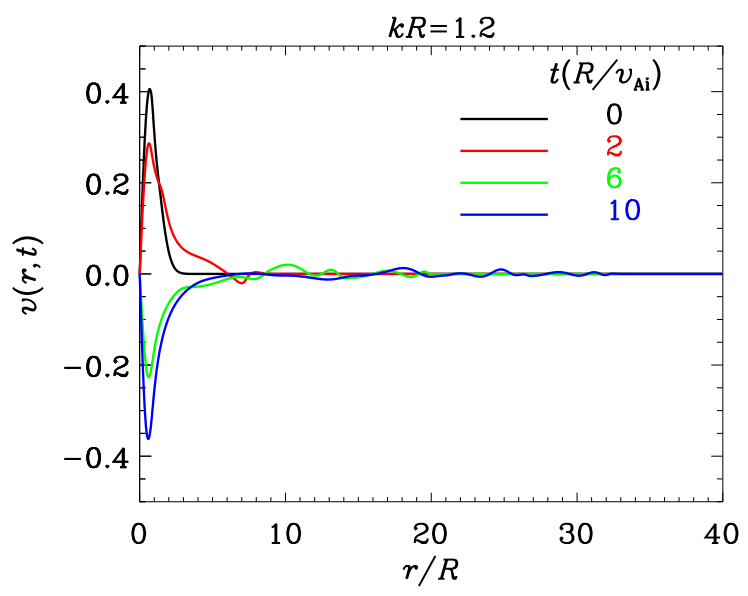

Figure 5. Spatial distribution of the transverse velocity perturbation at a number of different times for $k R=1.2$. The initial perturbation is described by Eq. (13). Here the density ratio $\rho_{\mathrm{i}} / \rho_{\mathrm{e}}=10$ and the steepness parameter $\mu=3$.

step-function density profiles by Terradas, Andries, and Goossens (2007), and for continuous profiles by Nakariakov, Hornsey, and Melnikov (2012); Chen et al. (2015a). To start, it is straightforward to derive an equation governing the transverse velocity perturbation $\delta v_{r}(r, z, t)$ from linearized, time-dependent, cold MHD equations. Formally expressing $\delta v_{r}(r, z, t)$ as $v(r, t) \sin (k z)$, one finds that $v(r, t)$ is governed by (e.g., Chen et al., 2015a)

$$
\frac{\partial^{2} v(r, t)}{\partial t^{2}}=v_{\mathrm{A}}^{2}(r)\left[\frac{\partial^{2}}{\partial r^{2}}+\frac{1}{r} \frac{\partial}{\partial r}-\left(\frac{1}{r^{2}}+k^{2}\right)\right] v(r, t) .
$$

With a $\rho(r)$ profile given by Eqs. (1) and (7), one readily evaluates the profile for the Alfvén speed $v_{\mathrm{A}}(r)=B / \sqrt{4 \pi \rho(r)}$. Equation (12) can then be readily solved when supplemented with appropriate initial and boundary conditions. For this purpose, we developed a simple finite-difference code second-order accurate in both space and time, and solve Eq. (12) on a uniform grid spanning $\left[0, r_{\text {outer }}\right]$ with a spacing $\Delta r=0.02 R$ and $r_{\text {outer }}=1000 R$. A uniform timestep $\Delta t=0.8 \Delta r / v_{\text {Ae }}$ is adopted to ensure numerical stability in view of the Courant condition. We have made sure that further refining the grid leads to no discernible difference. Furthermore, the outer boundary $r_{\text {outer }}$ is placed sufficiently far from the cylinder such that the signals to be analyzed are not contaminated by the perturbations reflected off the outer boundary. Pertinent to sausage modes, we require that $v(r=0, t)=0$. In addition, $v\left(r=r_{\text {outer }}, t\right)$ is specified to be zero for simplicity. Throughout this section, we examine a density ratio $\rho_{\mathrm{i}} / \rho_{\mathrm{e}}$ of 10 , and a steepness parameter $\mu$ of 3 . Moreover, for all computations we adopt the same initial condition (IC)

$$
v(r, t=0)=\frac{r}{R} \exp \left[-\left(\frac{r}{R}\right)^{2}\right], \quad \frac{\partial}{\partial t} v(r, t=0)=0
$$




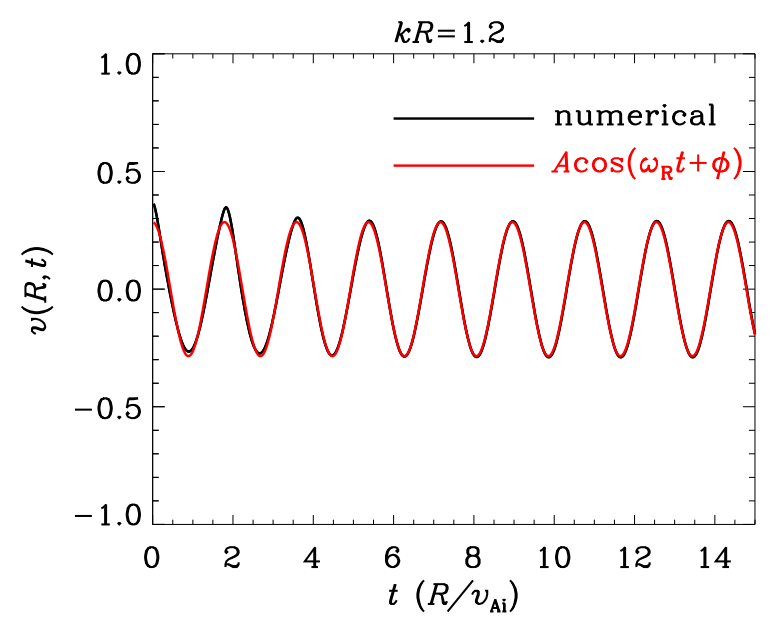

Figure 6. Temporal evolution of the transverse velocity perturbation $v(r=R, t)$ for $k R=1.2$. The initial perturbation is described by Eq. (13). In addition to the numerical result from this time-dependent computation (the black curve), a fit to this curve in the form $A \cos \left(\omega_{\mathrm{R}} t+\phi\right)$ is given by the red line for comparison. Here the density ratio $\rho_{\mathrm{i}} / \rho_{\mathrm{e}}=10$ and the steepness parameter $\mu=3$.

which is chosen not to be too localized to avoid exciting higher order modes.

Figure 5 presents the spatial distribution of $v(r, t)$ for $k R=1.2$ at a number of $t$ as labeled. One sees that as time progresses, some ripples propagate outward with the external Alfvén speed $\left(v_{\mathrm{Ae}}=\sqrt{\rho_{\mathrm{i}} / \rho_{\mathrm{e}}} v_{\mathrm{Ai}}=\sqrt{10} v_{\mathrm{Ai}}\right)$. The amplitudes of these ripples are rather insignificant and decrease with time, meaning that little energy is transmitted into the external medium for the adopted IC even though it is not an exact eigen-function. The majority of the energy is trapped in the cylinder, a signature of trapped modes.

That this computation pertains to the trapped regime is better shown by Fig. 6, where the temporal evolution of $v(R, t)$ is displayed. In addition to the numerical results (the black curve), a fit in the form $A \cos \left(\omega_{\mathrm{R}} t+\phi\right)$ is given by the red line. This fitting procedure yields that $\omega_{\mathrm{R}}=3.51 v_{\mathrm{Ai}} / R$, in exact agreement with the value found from solving the dispersion relation (Eq. 3). One sees that the black curve can be hardly told apart from the red one when $t \gtrsim 4 R / v_{\mathrm{Ai}}$, meaning that the signal at this location rapidly evolves into a trapped eigenmode.

What happens for a small $k R$ ? This is examined in Fig. 7 where the spatial dependence of $v(r, t)$ for $k R=0.1$ is presented. In response to the initial perturbation, some ripples are also seen to propagate away from the cylinder. However, in this case the signal close to the cylinder axis $(r=0)$ decays so rapidly that a different scale has to be used to plot $v(r, t)$ at large times (Fig. 7b). From Fig. $7 \mathrm{~b}$ one also sees that at a given time, the amplitude of the perturbations in the external medium tends to increase with distance first before decreasing towards the front (also see the blue curve in Fig. [7a). This is a signature of leaky eigen-functions (e.g., Cally, 1986; Terradas, Andries, and Goossens, 2007). 

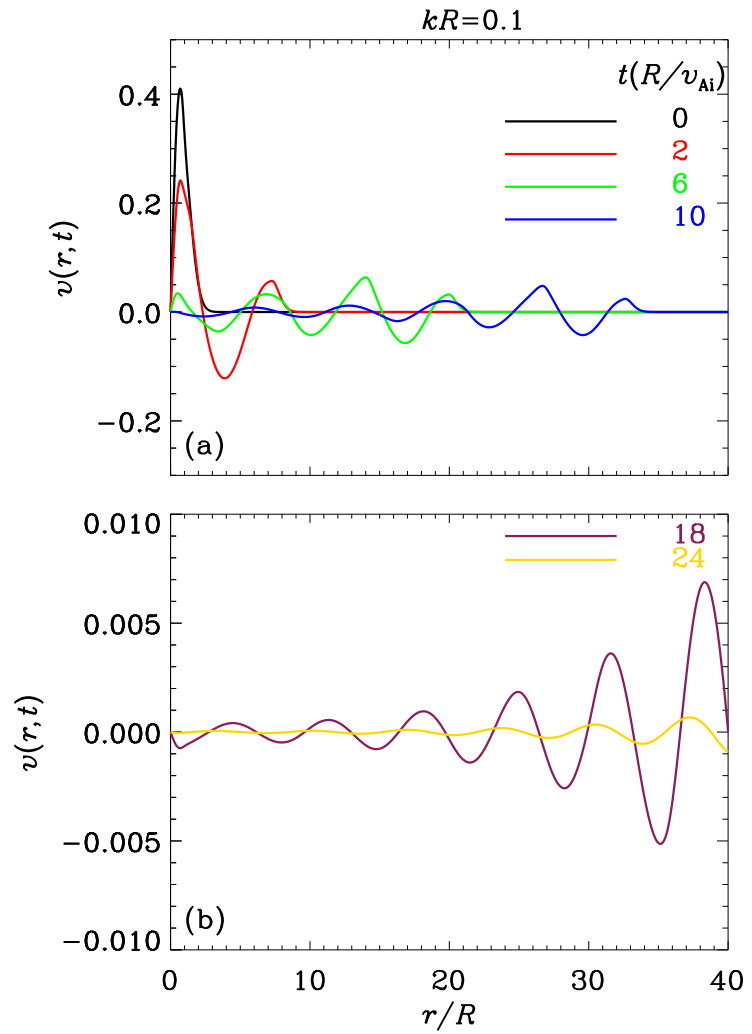

Figure 7. Similar to Fig. 5 but for $k R=0.1$. Note that the spatial distributions for $t=18$ and $24 R / v_{\mathrm{Ai}}$ are plotted in a separate panel.

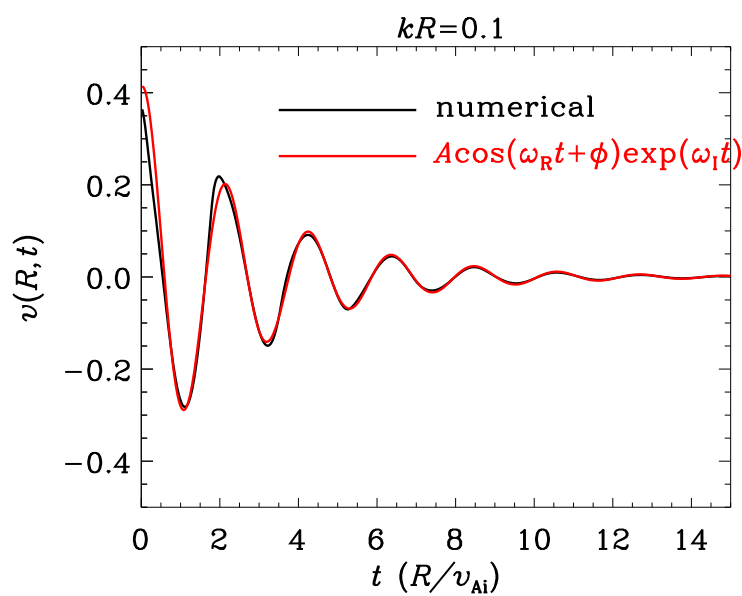

Figure 8. Similar to Fig. 6 but for $k R=0.1$. Note that the fit (the red curve) to the time-dependent solution (black) is in the form $A \cos \left(\omega_{\mathrm{R}} t+\phi\right) \exp \left(\omega_{\mathrm{I}} t\right)$. 
Figure 8 presents the temporal evolution of $v(R, t)$ (the black curve) together with a fitting in the form $A \cos \left(\omega_{\mathrm{R}} t+\phi\right) \exp \left(\omega_{\mathrm{I}} t\right)$ (red). From this fitting we find that $\left[\omega_{\mathrm{R}}, \omega_{\mathrm{I}}\right]=[2.98,-0.34] v_{\mathrm{Ai}} / R$, coinciding with the eigenmode computation for the adopted $k R$. One sees that the black and red curves agree closely with each other for $t \gtrsim 2.5 R / v_{\mathrm{Ai}}$, substantiating the interpretation that the signal settles to a leaky eigenmode.

\section{B. Fast kink modes in nonuniform cylinders: a resistive, linear MHD computation}

This section provides some details for the resistive, linear MHD computations that we employ to establish the functions $F_{\text {kink }}$ and $H_{\text {kink }}$ contained in Eqs. (10) and (11). Such a description seems informative even though our approach is identical to the one adopted by Terradas, Oliver, and Ballester (2006, hereafter TOB06), since the density profile given by Eq. (1) has not been explored for resonantly damped kink modes. Now that the approach has been detailed in section 3.1 in TOB06, it suffices to note here that we are looking for a dimensionless complex-valued angular frequency $\omega_{\mathrm{kink}} L / v_{\mathrm{Ai}}$ for a set of dimensionless parameters $\left[L / R, \mu, \rho_{\mathrm{i}} / \rho_{\mathrm{e}}\right]$. The magnetic Reynolds number $R_{\mathrm{m}}=v_{\mathrm{Ai}} R / \bar{\eta}$ is also relevant, where $\bar{\eta}$ is the resistivity and assumed to be uniform.

Figure 9 presents the $R_{\mathrm{m}}$ dependence of the real $\left(\omega_{\mathrm{R}}\right)$ and imaginary $\left(\omega_{\mathrm{I}}\right)$ parts of the dimensionless angular frequency for $k R=0.1 \pi$ and $\rho_{\mathrm{i}} / \rho_{\mathrm{e}}=20$. A number of $\mu$ values are examined as given by the curves in different colors. From Fig. 9b one sees that the curves show a significant $R_{\mathrm{m}}$ dependence only for relatively small $R_{\mathrm{m}}$. As discussed in TOB06, this is attributable to the competition between resistivity and resonant absorption in damping the kink modes. For large (small) $R_{\mathrm{m}}$, resonant absorption (resistivity) plays a more important role and consequently the damping rate $\left|\omega_{I}\right|$ is insensitive (sensitive) to $R_{\mathrm{m}}$. Interestingly, in agreement with Fig. 2 of TOB06, $\left|\omega_{\mathrm{I}}\right|$ is not sensitive to the density profile steepness when $R_{\mathrm{m}}$ is small. On the other hand, $\left|\omega_{\mathrm{I}}\right|$ rapidly settles to some asymptotic value when $R_{\mathrm{m}}$ exceeds some critical value. Similar to TOB06, with increasing profile steepness this critical $R_{\mathrm{m}}$ increases, whereas the asymptotic $\left|\omega_{\mathrm{I}}\right|$ decreases. Examining Fig. 9k, one finds that $\omega_{\mathrm{R}}$ is different for different $\mu$ values even at small $R_{\mathrm{m}}$. Despite this, what is important for the present purpose is that neither $\omega_{\mathrm{R}}$ nor $\omega_{\mathrm{I}}$ depends on $R_{\mathrm{m}}$ when $R_{\mathrm{m}}$ is sufficiently large. And their asymptotic values are taken to be the eigen-frequencies of kink modes whose damping is solely due to resonant absorption.

How do these saturation values depend on the steepness parameter $\mu$ when the rest of the parameters are fixed? This is examined in Fig. 10 where a series of computations are conducted for a number of density ratios $\rho_{\mathrm{i}} / \rho_{\mathrm{e}}$ as labeled. The dimensionless longitudinal wavenumber $k R$ is also taken to be $0.1 \pi$. For presentation purposes, both $\omega_{\mathrm{R}}$ and $\omega_{\mathrm{I}}$ are normalized to the kink frequency $\omega_{k}$, which is attained for density profiles of a step-function form. It turns out that the correction to $\omega_{k}$ due to dispersion is not negligible at the chosen $k R$, meaning that $\omega_{k}$ needs to be computed by solving the relevant dispersion relation (e.g., Edwin and Roberts, 1983, Eq. 8b). The computed value is a few percent different 

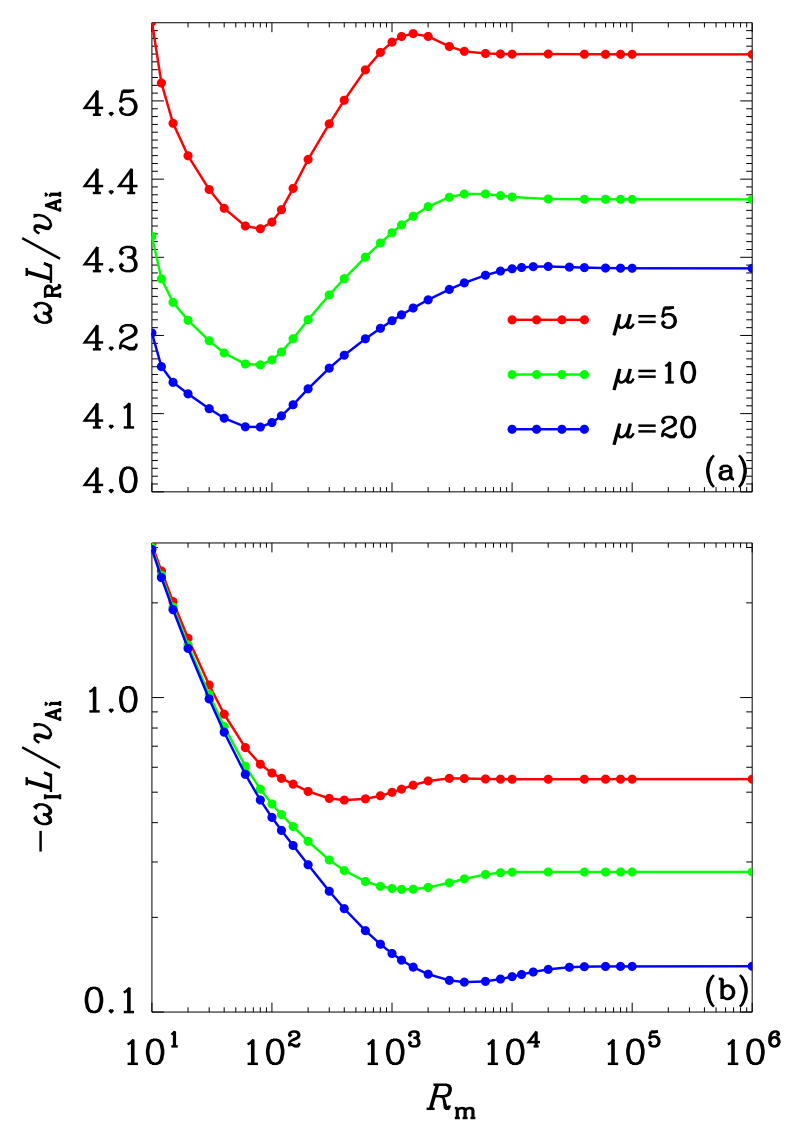

Figure 9. Dependence on the magnetic Reynolds number $R_{\mathrm{m}}$ of (a) the real and (b) imaginary parts of the dimensionless eigen-frequency for kink modes in cylinders with a transverse density profile given by Eqs. (1) and (7). A number of different steepness parameters $\mu$ are examined as labeled. Here the dimensionless longitudinal wavenumber $k R=0.1 \pi$, and the density ratio $\rho_{\mathrm{i}} / \rho_{\mathrm{e}}$ is fixed at 20 .

from its thin-tube counterpart, namely $\sqrt{2} k v_{\mathrm{Ai}} / \sqrt{1+\rho_{\mathrm{e}} / \rho_{\mathrm{i}}}$ (e.g., Eq. 40 in Soler et al., 2013, hereafter S13). Similar to Fig. 1 in S13, our Fig. 10] shows that $\omega_{\mathrm{R}}$ and $\left|\omega_{I}\right|$ tend to decrease with increasing density profile steepness, approaching the step-function values when $\mu$ is sufficiently large. In addition, $\left|\omega_{\mathrm{I}}\right| / \omega_{k}$ at a fixed steepness parameter increases with increasing $\rho_{\mathrm{i}} / \rho_{\mathrm{e}}$. However, while here $\omega_{\mathrm{R}} / \omega_{k}$ tends to increase with $\rho_{\mathrm{i}} / \rho_{\mathrm{e}}$ regardless of $\mu$, it does not show a monotonical dependence on $\rho_{\mathrm{i}} / \rho_{\mathrm{e}}$ in Fig. 1 of S13 when the steepness parameter is fixed. This difference signifies the importance of the detailed description of the density profile in determining the properties of resonantly damped kink modes: while a $\mu$-power profile is examined here, S13 explored a sine profile (Eq. 63 therein). 

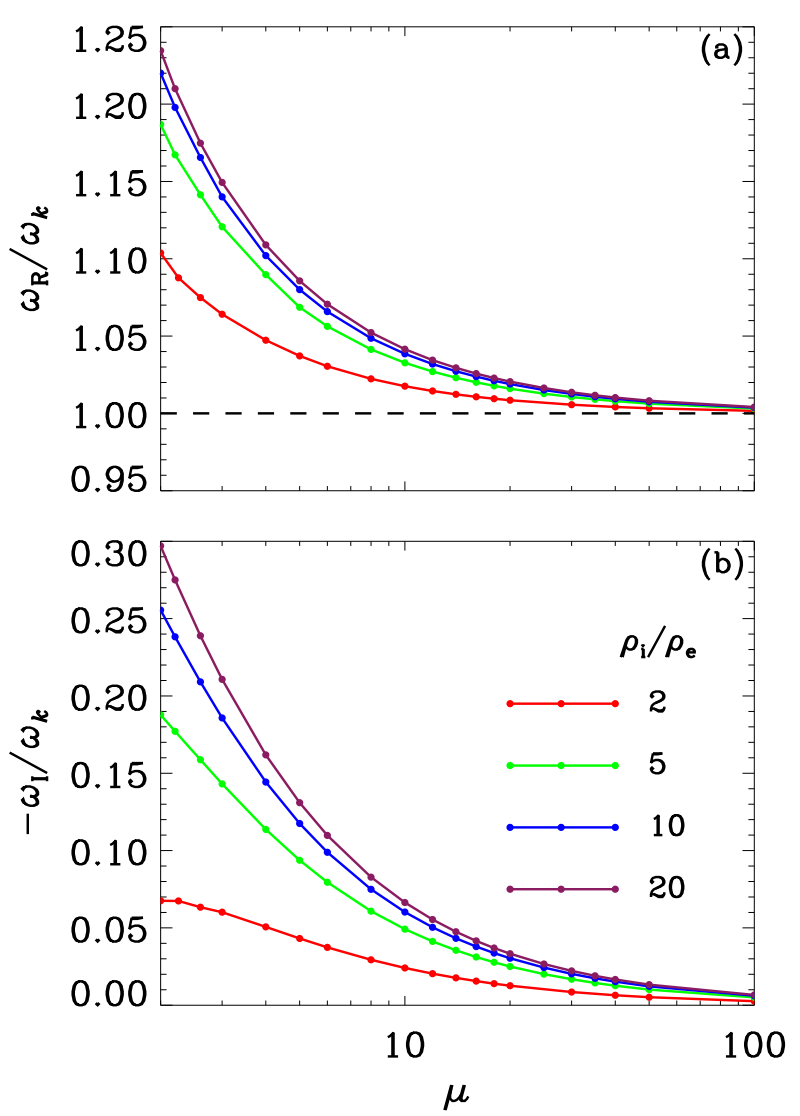

Figure 10. Dependence on the steepness parameter $\mu$ of (a) the real and (b) imaginary parts of the eigen-frequencies for resonantly damped kink modes in cylinders with a transverse density profile given by Eqs. (1) and (7). A number of density ratios $\rho_{\mathrm{i}} / \rho_{\mathrm{e}}$ are examined as labeled. Here the dimensionless longitudinal wavenumber $k R=0.1 \pi$. The eigen-frequencies are found with linear resistive MHD computations at sufficiently large magnetic Reynolds numbers. They are normalized to $\omega_{k}$, the value attained for density profiles of a step-function form. See text for details.

\section{Examining sausage modes in nonuniform cylinders with series-expansion-based methods}

So far two series-expansion-based methods have been available to derive explicit expressions for the sausage perturbations in the nonuniform portion of the density distribution. One (approach I, S13) is based on singular expansions as a byproduct of a comprehensive examination of resonantly damped kink modes, whereas the other (approach II, paper I) is based on regular expansions. This section provides a rather detailed comparison between the two.

To facilitate this comparison, let us focus on density profiles considered by both studies, where a transition layer (TL) connects a uniform cord (with density $\rho_{\mathrm{i}}$ ) and a uniform external medium (with density $\rho_{\mathrm{e}}$ ). The TL is of width $l$ and centers around $r=R$. Approach I solves the perturbation equation (Eq. 4 in 
S13) by conducting an expansion about the Alfvén resonance $r_{\mathrm{A}}$ where $\omega_{\mathrm{R}}=$ $k v_{\mathrm{A}}$. For the density profiles in question, $r_{\mathrm{A}}$ is located in the TL. Valid for arbitrary azimuthal wavenumbers $m$, the analysis in S13 yields that for sausage modes $(m=0)$, the series solutions are regular even though $r_{\mathrm{A}}$ is a regular singular point. Physically, this means that sausage modes do not resonantly couple to the Alfvén continuum. Approach II capitalizes on this fact and solves the perturbation equation (Eq. 6 in paper I) by performing a regular series expansion about $r=R$. In this aspect, approach II is equivalent to I and both should yield identical solutions, provided that a point exists in the TL such that $\omega_{\mathrm{R}}=k v_{\mathrm{A}}$. Let $r_{\mathrm{A}}$ denote this point for brevity, although it is not a resonance for sausage modes.

Before proceeding, we note that the perturbations in the external medium were required to be evanescent by S13, since leaky modes were not of interest therein. Consequently, the Fourier amplitude of the Eulerian perturbation of total pressure was expressed with $K_{0}\left(k_{\perp, \mathrm{e}} r\right)$, the modified Bessel function of the first kind (Eq. 10 in S13). To account for leaky sausage modes, this needs to be replaced with $H_{0}^{(1)}\left(\mu_{\mathrm{e}} r\right)$ (e.g., Cally, 1986). Here $\mu_{\mathrm{e}}$ is defined by Eq. (4), and by definition $\mu_{\mathrm{e}}^{2}=-k_{\perp, \mathrm{e}}^{2}$. With the notations in S13, the DR therein then reads

$$
\begin{aligned}
& \frac{-\mu_{\mathrm{e}}}{\rho_{\mathrm{e}}\left(\omega^{2}-k^{2} v_{\mathrm{A}, \mathrm{e}}^{2}\right)} \frac{H_{1}^{(1)}\left[\mu_{\mathrm{e}}(R+l / 2)\right]}{H_{0}^{(1)}\left[\mu_{\mathrm{e}}(R+l / 2)\right]} \mathcal{G}_{\mathrm{e}}-\Xi_{\mathrm{e}} \\
& \frac{-\mu_{\mathrm{e}}}{\rho_{\mathrm{e}}\left(\omega^{2}-k^{2} v_{\mathrm{A}, \mathrm{e}}^{2}\right)} \frac{H_{1}^{(1)}\left[\mu_{\mathrm{e}}(R+l / 2)\right]}{H_{0}^{(1)}\left[\mu_{\mathrm{e}}(R+l / 2)\right]} \mathcal{F}_{\mathrm{e}}-\Gamma_{\mathrm{e}} \\
& -\frac{-k_{\perp, \mathrm{i}}}{\rho_{\mathrm{i}}\left(\omega^{2}-k^{2} v_{\mathrm{A}, \mathrm{i}}^{2}\right)} \frac{J_{1}\left[k_{\perp, \mathrm{i}}(R-l / 2)\right]}{J_{0}\left[k_{\perp, \mathrm{i}}(R-l / 2)\right]} \mathcal{G}_{\mathrm{i}}-\Xi_{\mathrm{i}} \\
& =0 .
\end{aligned}
$$

For non-leaky waves, this recovers Eq. (27) in S13, given that

$$
\frac{\mu_{\mathrm{e}} H_{1}^{(1)}\left[\mu_{\mathrm{e}}(R+l / 2)\right]}{H_{0}^{(1)}\left[\mu_{\mathrm{e}}(R+l / 2)\right]}=\frac{k_{\perp, \mathrm{e}} K_{1}\left[k_{\perp, \mathrm{e}}(R+l / 2)\right]}{K_{0}\left[k_{\perp, \mathrm{e}}(R+l / 2)\right]},
$$

where we have used the relation (p. 375 in Abramowitz and Stegun, 1970)

$$
K_{m}(w)=\frac{\pi}{2} i^{m+1} H_{m}^{(1)}(i w) \quad\left(-\pi<\arg w \leq \frac{\pi}{2}, m=0,1, \cdots\right) .
$$

For illustration purposes, in what follows we examine a linear profile for the density distribution in the TL (Eq. 4 in paper I), and assume that $\rho_{\mathrm{i}} / \rho_{\mathrm{e}}=100$. For approach I, we solve Eq. (14) instead of Eq. (27) in S13, and for approach II we solve our Eq. (17) in paper I. 


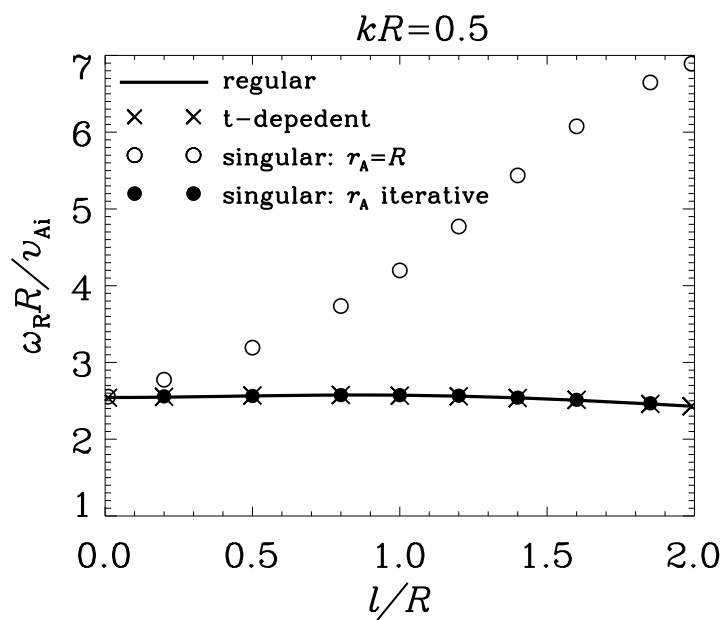

Figure 11. Comparison of two series-expansion-based methods for computing eigen-frequencies of sausage modes. To facilitate this comparison, the density distribution is different from the one described by Eq. (1). Instead, the profile labeled "linear" in Eq. (4) of paper I is adopted. Here the dimensionless longitudinal wavenumber $k R=0.5$, pertinent to the trapped regime $\left(\omega_{\mathrm{I}}=0\right)$ for the chosen density ratio $\rho_{\mathrm{i}} / \rho_{\mathrm{e}}$ of 100 . The real part of the eigen-frequency $\omega_{\mathrm{R}}$ is displayed as a function of $l / R$, the density lengthscale in units of loop radius. The black curve represents the results found with the method based on regular expansions, and the crosses represent those obtained with analyzing the perturbation signals in the corresponding time-dependent computations. Two treatments are adopted for the method based on singular expansions. In one treatment the location of the nominal Alfvén resonance $r_{\mathrm{A}}$ is supposed to be $R$ (the open dots), while in the other it is found iteratively (filled). See text for details.

Figure 11 compares the eigen-frequencies found for a series of $l / R$ from the two approaches when $k R$ equals 0.5 . This $k R$ falls into the trapped regime since the imaginary parts $\left(\omega_{\mathrm{I}}\right)$ of the eigen-frequencies are zero, and hence only the real parts $\left(\omega_{\mathrm{R}}\right)$ are shown. The solid line labeled "regular" represents the solutions from approach II. When adopting approach I, we examine two different treatments for the location of $r_{\mathrm{A}}$ : in one we simply take $r_{\mathrm{A}}$ to be $R$ (the open dots), whereas in the other we solve Eq. (14) iteratively to simultaneous derive $\omega_{\mathrm{R}}$ and $r_{\mathrm{A}}$ (the filled dots). Note that a value for $r_{\mathrm{A}}$ needs to be specified before solving this DR. However, the Alfvén speed at this guessed location usually does not equal to $\omega_{\mathrm{R}} / k$ thus found. Hence this latter iterative treatment. One sees that the filled dots fall on the solid curve, and both are in exact agreement with the crosses representing the values found from fitting the signals $v(R, t)$ in the corresponding time-dependent computations (see Appendix A). This means that both approaches yield correct results, and approach II can be seen as a specific case of the more general analysis presented in S13. Nonetheless, the advantage of approach II is that there is no need to find $r_{\mathrm{A}}$, which is necessary for approach $\mathrm{I}$, given that simply assuming $r_{\mathrm{A}}=R$ beforehand can yield considerably different results (see the open dots).

Some considerable difference arises when leaky sausage modes are examined. Let us consider $k R=0.01$, for which the real $\left(\omega_{\mathrm{R}}\right)$ and imaginary $\left(\omega_{\mathrm{I}}\right)$ parts of 

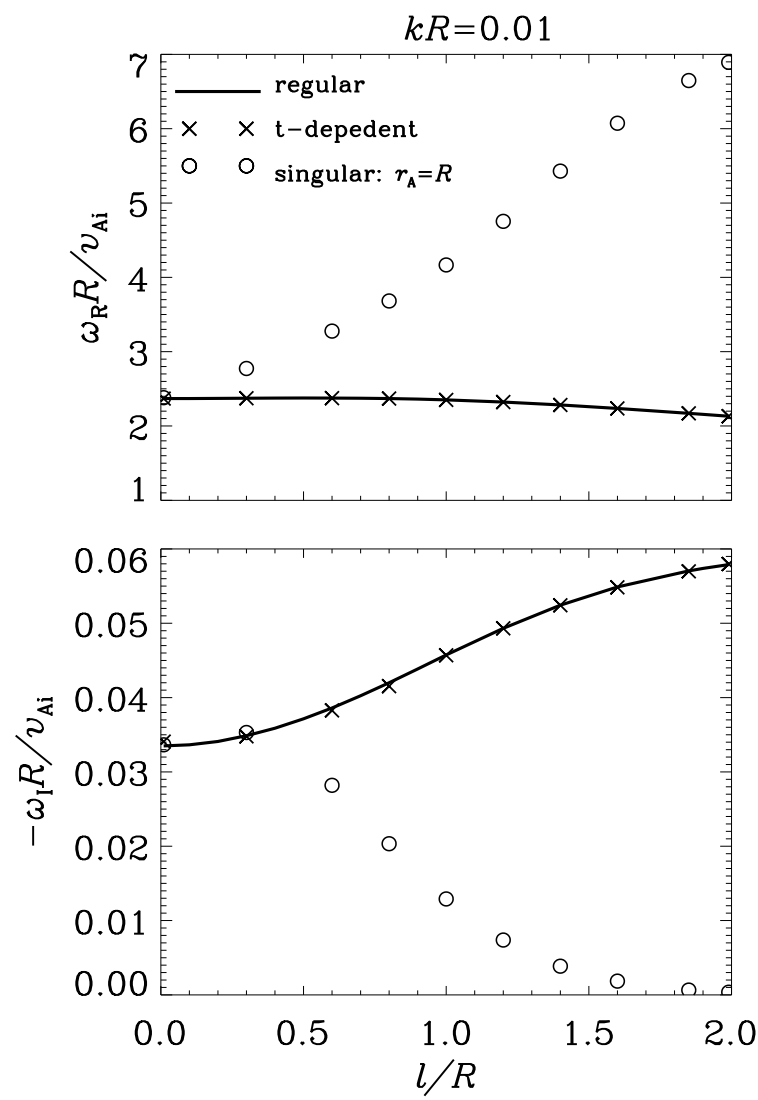

Figure 12. Similar to Fig. 11 but for $k R=0.01$ pertinent to leaky modes, for which $\omega_{\mathrm{I}}$ is non-zero and displayed in a separate panel. See text for details.

the complex-valued eigen-frequencies are presented in Fig. 12, The results from approach II are given by the solid line, and are found to agree well with the values found from analyzing the time-dependent results (the crosses). However, they differ substantially from the results found with approach I where we assume that $r_{\mathrm{A}}=R$. In this case the iterative treatment does not work because no point in the TL corresponds to a $v_{\mathrm{A}}$ that equals $\omega_{\mathrm{R}} / k$. The reason is that in the leaky regime, the apparent phase speed $\omega_{\mathrm{R}} / k$ consistently exceeds $v_{\mathrm{Ae}}$, which in turn always exceeds the Alfvén speeds in the TL.

Despite the afore-mentioned discussions, we stress that the mathematical approach presented in S13 is sufficiently general to treat modes with arbitrary azimuthal wavenumber $m$, trapped sausage modes $(m=0)$ included. A singular series expansion is necessary for treating all modes with $m \neq 0$.

\section{References}

Abramowitz, M., Stegun, I.A.: 1970, Handbook of mathematical functions : with formulas, 
graphs, and mathematical tables, U.S. Dept. of Commerce, National Bureau of Standards, Washington, D.C.ADS [1970hmfw.book...... A]

Andries, J., van Doorsselaere, T., Roberts, B., Verth, G., Verwichte, E., Erdélyi, R.: 2009, Coronal Seismology by Means of Kink Oscillation Overtones. Space Sci. Rev. 149, 3. DOI ADS [2009SSRv..149...3A]

Arregui, I., Asensio Ramos, A., Pascoe, D.J.: 2013, Determination of Transverse Density Structuring from Propagating Magnetohydrodynamic Waves in the Solar Atmosphere. Astrophys. J. Lett. 769, L34. DOI ADS [2013ApJ ...769L..34A]

Arregui, I., Oliver, R., Ballester, J.L.: 2012, Prominence Oscillations. Living Reviews in Solar Physics 9, 2. DOI ADS [2012LRSP.......2A]

Arregui, I., Soler, R., Asensio Ramos, A.: 2015, Model Comparison for the Density Structure across Solar Coronal Waveguides. Astrophys. J. 811, 104. DOI ADS [2015ApJ . . .811 . 104A]

Arregui, I., Andries, J., Van Doorsselaere, T., Goossens, M., Poedts, S.: 2007, MHD seismology of coronal loops using the period and damping of quasi-mode kink oscillations. Astron. Astrophys. 463, 333. DOI ADS. [2007A\&A...463..333A]

Aschwanden, M.J., Nakariakov, V.M., Melnikov, V.F.: 2004, Magnetohydrodynamic Sausage-Mode Oscillations in Coronal Loops. Astrophys. J. 600, 458. DOI ADS [2004ApJ...600..458A]

Aschwanden, M.J., Fletcher, L., Schrijver, C.J., Alexander, D.: 1999, Coronal Loop Oscillations Observed with the Transition Region and Coronal Explorer. Astrophys. J. 520, 880. DOI ADS [1999ApJ...520..880A]

Asensio Ramos, A., Arregui, I.: 2013, Coronal loop physical parameters from the analysis of multiple observed transverse oscillations. Astron. Astrophys. 554, A7. DOI ADS [2013A\&A...554A...7A]

Banerjee, D., Erdélyi, R., Oliver, R., O'Shea, E.: 2007, Present and Future Observing Trends in Atmospheric Magnetoseismology. Solar Phys. 246, 3. DOI ADS [2007SoPh..246....3B]

Cally, P.S.: 1986, Leaky and non-leaky oscillations in magnetic flux tubes. Solar Phys. 103, 277. DOI ADS [1986SoPh..103..277C]

Chen, S.-X., Li, B., Xia, L.-D., Yu, H.: 2015a, Periods and Damping Rates of Fast Sausage Oscillations in Multishelled Coronal Loops. Solar Phys. 290, 2231. DOI. ADS [2015SoPh..290.2231C]

Chen, S.-X., Li, B., Xiong, M., Yu, H., Guo, M.-Z.: 2015b, Standing Sausage Modes in Nonuniform Magnetic Tubes: An Inversion Scheme for Inferring Flare Loop Parameters. Astrophys. J. 812, 22. DOI ADS [2015ApJ ...812...22C]

De Moortel, I., Nakariakov, V.M.: 2012, Magnetohydrodynamic waves and coronal seismology: an overview of recent results. Philosophical Transactions of the Royal Society of London Series A 370, 3193. DOI ADS [2012RSPTA.370.3193D]

Edwin, P.M., Roberts, B.: 1983, Wave propagation in a magnetic cylinder. Solar Phys. 88, 179. DOI ADS [1983SoPh...88..179E]

Edwin, P.M., Roberts, B.: 1988, Employing analogies for ducted MHD waves in dense coronal structures. Astron. Astrophys. 192, 343. ADS [1988A\&A...192..343E]

Goossens, M., Andries, J., Aschwanden, M.J.: 2002, Coronal loop oscillations. An interpretation in terms of resonant absorption of quasi-mode kink oscillations. Astron. Astrophys. 394, L39. DOI ADS [2002A\&A ...394L..39G]

Goossens, M., Arregui, I., Ballester, J.L., Wang, T.J.: 2008, Analytic approximate seismology of transversely oscillating coronal loops. Astron. Astrophys. 484, 851. DOI ADS [2008A\&A. . 484.851G]

Grant, S.D.T., Jess, D.B., Moreels, M.G., Morton, R.J., Christian, D.J., Giagkiozis, I., Verth, G., Fedun, V., Keys, P.H., Van Doorsselaere, T., Erdélyi, R.: 2015, Wave Damping Observed in Upwardly Propagating Sausage-mode Oscillations Contained within a Magnetic Pore. Astrophys. J. 806, 132. DOI ADS [2015ApJ...806..132G]

Heyvaerts, J., Priest, E.R.: 1983, Coronal heating by phase-mixed shear Alfven waves. Astron. Astrophys. 117, 220. ADS [1983A\&A...117..220H]

Hollweg, J.V., Yang, G.: 1988, Resonance absorption of compressible magnetohydrodynamic waves at thin 'surfaces'. J. Geophys. Res. 93, 5423. DOI ADS [1988JGR... 93.5423H]

Jess, D.B., Mathioudakis, M., Erdélyi, R., Crockett, P.J., Keenan, F.P., Christian, D.J.: 2009, Alfvén Waves in the Lower Solar Atmosphere. Science 323. DOI. ADS [2009Sci...323.1582J] 
Kolotkov, D.Y., Nakariakov, V.M., Kupriyanova, E.G., Ratcliffe, H., Shibasaki, K.: 2015, Multi-mode quasi-periodic pulsations in a solar flare. Astron. Astrophys. 574, A53. DOI ADS [2015A\&A...574A..53K]

Kopylova, Y.G., Melnikov, A.V., Stepanov, A.V., Tsap, Y.T., Goldvarg, T.B.: 2007, Oscillations of coronal loops and second pulsations of solar radio emission. Astronomy Letters $\mathbf{3 3}$, 706. DOI ADS [2007AstL...33..706K]

Kupriyanova, E.G., Melnikov, V.F., Shibasaki, K.: 2013, Spatially Resolved Microwave Observations of Multiple Periodicities in a Flaring Loop. Solar Phys. 284, 559. DOI ADS [2013SoPh. .284..559K]

Liu, W., Ofman, L.: 2014, Advances in Observing Various Coronal EUV Waves in the SDO Era and Their Seismological Applications (Invited Review). Solar Phys. 289, 3233. DOI ADS [2014SoPh..289.3233L]

Lopin, I., Nagorny, I.: 2014, Fast-sausage oscillations in coronal loops with smooth boundary. Astron. Astrophys. 572, A60. DOI ADS [2014A\&A...572A..60L]

Lopin, I., Nagorny, I.: 2015, Sausage Waves in Transversely Nonuniform Monolithic Coronal Tubes. Astrophys. J. 810, 87. DOI ADS [2015ApJ ..810...87L]

McLean, D.J., Sheridan, K.V.: 1973, A Damped Train of Regular Metre-Wave Pulses from the Sun. Solar Phys. 32, 485. DOI ADS [1973SoPh...32..485M]

Meerson, B.I., Sasorov, P.V., Stepanov, A.V.: 1978, Pulsations of type IV solar radio emission - The bounce-resonance effects. Solar Phys. 58, 165. DOI ADS [1978SoPh...58...165M]

Moreels, M.G., Van Doorsselaere, T., Grant, S.D.T., Jess, D.B., Goossens, M.: 2015, Energy and energy flux in axisymmetric slow and fast waves. Astron. Astrophys. 578, A60. DOI ADS [2015A\&A...578A.60M]

Morton, R.J., Erdélyi, R., Jess, D.B., Mathioudakis, M.: 2011, Observations of Sausage Modes in Magnetic Pores. Astrophys. J. Lett. 729, L18. DOI ADS [2011ApJ...729L..18M]

Morton, R.J., Verth, G., Jess, D.B., Kuridze, D., Ruderman, M.S., Mathioudakis, M., Erdélyi, R.: 2012, Observations of ubiquitous compressive waves in the Sun's chromosphere. Nature Communications 3, 1315. DOI ADS [2012NatCo...3E1315M]

Nakariakov, V.M., Melnikov, V.F.: 2009, Quasi-Periodic Pulsations in Solar Flares. Space Sci. Rev. 149, 119. DOI ADS [2009SSRv ..149..119N]

Nakariakov, V.M., Verwichte, E.: 2005, Coronal Waves and Oscillations. Living Reviews in Solar Physics 2, 3. DOI ADS [2005LRSP........3N]

Nakariakov, V.M., Hornsey, C., Melnikov, V.F.: 2012, Sausage Oscillations of Coronal Plasma Structures. Astrophys. J. 761, 134. DOI ADS [2012ApJ . .761..134N]

Nakariakov, V.M., Melnikov, V.F., Reznikova, V.E.: 2003, Global sausage modes of coronal loops. Astron. Astrophys. 412, L7. DOI ADS. [2003A\&A . .412L ...7N]

Nakariakov, V.M., Ofman, L., Deluca, E.E., Roberts, B., Davila, J.M.: 1999, TRACE observation of damped coronal loop oscillations: Implications for coronal heating. Science $\mathbf{2 8 5}$, 862. DOI ADS [1999Sci...285..862N]

Roberts, B.: 2008, Progress in coronal seismology. In: Erdélyi, R., Mendoza-Briceno, C.A. (eds.) IAU Symposium, IAU Symposium 247, 3. DOI ADS [2008IAUS..247....3R]

Roberts, B., Edwin, P.M., Benz, A.O.: 1984, On coronal oscillations. Astrophys. J. 279, 857. DOI ADS [1984ApJ...279. .857R]

Rosenberg, H.: 1970, Evidence for MHD Pulsations in the Solar Corona. Astron. Astrophys. 9, 159. ADS [1970A\&A.......159R]

Ruderman, M.S., Roberts, B.: 2002, The Damping of Coronal Loop Oscillations. Astrophys. J. 577, 475. DOI ADS [2002ApJ ...577..475R]

Soler, R., Goossens, M., Terradas, J., Oliver, R.: 2013, The Behavior of Transverse Waves in Nonuniform Solar Flux Tubes. I. Comparison of Ideal and Resistive Results. Astrophys. J. 777, 158. DOI ADS [2013ApJ ...777..158S]

Soler, R., Goossens, M., Terradas, J., Oliver, R.: 2014, The Behavior of Transverse Waves in Nonuniform Solar Flux Tubes. II. Implications for Coronal Loop Seismology. Astrophys. $J .781,111$. DOI ADS [2014ApJ ...781..111S]

Spruit, H.C.: 1982, Propagation speeds and acoustic damping of waves in magnetic flux tubes. Solar Phys. 75, 3. DOI ADS [1982SoPh...75 ...3S]

Terradas, J., Andries, J., Goossens, M.: 2007, On the Excitation of Leaky Modes in Cylindrical Loops. Solar Phys. 246, 231. DOI. ADS. [2007SoPh..246..231T]

Terradas, J., Oliver, R., Ballester, J.L.: 2006, Damped Coronal Loop Oscillations: Timedependent Results. Astrophys. J. 642, 533. DOI ADS. [2006ApJ...642..533T]

Tomczyk, S., McIntosh, S.W.: 2009, Time-Distance Seismology of the Solar Corona with CoMP. Astrophys. J. 697, 1384. DOI ADS [2009ApJ...697.1384T] 
Uchida, Y.: 1970, Diagnosis of Coronal Magnetic Structure by Flare-Associated Hydromagnetic Disturbances. Pub. Astron. Soc. Japan 22, 341. ADS [1970PASJ...22..341U]

Vasheghani Farahani, S., Hornsey, C., Van Doorsselaere, T., Goossens, M.: 2014, Frequency and Damping Rate of Fast Sausage Waves. Astrophys. J. 781, 92. DOI ADS [2014ApJ ...781...92V]

Zajtsev, V.V., Stepanov, A.V.: 1975, On the origin of pulsations of type IV solar radio emission. Plasma cylinder oscillations (I). Issledovaniia Geomagnetizmu Aeronomii i Fizike Solntsa 37, 3. ADS [1975IGAFS..37...3Z]

Zaqarashvili, T.V., Erdélyi, R.: 2009, Oscillations and Waves in Solar Spicules. Space Sci. Rev. 149, 355. DOI ADS [2009SSRv..149..355Z] 